International Journal of Modern Physics E

(C) World Scientific Publishing Company

\title{
High-spin structures as the probes of proton-neutron pairing
}

\author{
A. V. Afanasjev \\ Department of Physics and Astronomy, Mississippi State University, Mississippi State, P.O. \\ Box 5167, MS 39762, USA \\ Received (received date) \\ Revised (revised date)
}

\begin{abstract}
Rotating $N=Z$ nuclei in the mass $A=58-80$ region have been studied within the framework of isovector mean field theory. Available data is well and systematically described in the calculations. The present study supports the presence of strong isovector $n p$ pair field at low spin, which is, however, destroyed at high spin. No clear evidence for the existence of the isoscalar $t=0 n p$ pairing has been found.
\end{abstract}

\section{Introduction}

It is well known that in the nuclei away from the $N=Z$ line proton-proton $(p p)$ and neutron-neutron $(n n)$ pairing dominate. In the $N \approx Z$ nuclei, protons and neutrons occupy the same levels. Strong $n p$ pair correlations are expected because of large spatial overlap of their wave functions. These correlations can be isoscalar and isovector. Figuring out their character and whether they form a static pair condensate (an average field) in respective channel has been a challenge since medium mass $N=Z$ nuclei have come into reach of experiment.

At present, the situation with the isovector $n p$-pairing is most clarified. The strength of the isovector $n p$-pairing is well defined by the isospin symmetry 1 . A number of experimental observables such as binding energies of the $T=0$ and $T=1$ states in even-even and odd-odd $N=Z$ nuclei 2314 , the observation of only one even-spin $T=0$ band in ${ }^{74} \mathrm{Rb}{ }^{4}$ instead of two nearly degenerate bands expected in the case of no $t=1 \mathrm{np}$-pairing $\mathrm{a}$ clearly point on the existence of pairing condensate in this channel. The analysis of pairing vibrations around ${ }^{56} \mathrm{Ni}$ indicates a collective behavior of the isovector pairing vibrations but does not support any appreciable collectivity in the isoscalar channel 516 .

On the other hand, it is still an issue of debate whether the isoscalar np-pair correlations lead to a pairing condensate. The calculations with the realistic forces (Paris force, Argonne V14 force) indicate that the isoscalar pairing gap in the symmetric nuclear matter is 3 times larger than the isovector one 7 . The potential

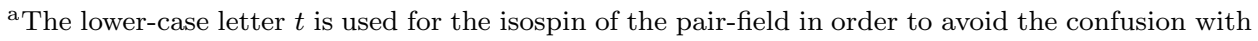
the total isospin of the states denoted by $T$. 
problem stems from the transition from realistic to effective interaction: the extremely strong $t=0$ pairing emerges essentially from the fact that with respect to the $t=1$ channel, dominated by the central force, the tensor force is acting additionally. However, the medium modification (screening) of the tensor force is still controversial subject 8 . Thus, the addition of tensor component into isoscalar pairing channel of the models based on effective forces may be necessary for a correct description of $n p$-pairing in this channel. In the existing mean-field models, this component is neglected. An additional challenge lies in the fact that the strength of the effective isoscalar $t=0 \mathrm{np}$-pairing is not known, and thus has to be defined by the comparison with experimental data. The quantity most frequently used for that is Wigner energy 9 , but it does not provide a unique and reliable way to define this strength (see discussion in Ref. 10).

In a given situation, two major questions arise, namely, (i) what are the physical observables which are sensitive to isoscalar np-pair condensate and (ii) which theoretical framework has to be used for the description of such systems?

Isoscalar $n p$-pairing may play a role in single-beta decay 1112 (see, however, Refs. 13144), double-beta decay 15/16, transfer reactions $17 / 18$ (see, however, Ref. 19), alpha decay and alpha correlations 20|21. However, since no symmetryunrestricted mean-field (and beyond mean field) calculations of $n p$-pairing, based on realistic effective interaction and the isospin-conserving formalism, have been carried out so far, no hard evidence for the elusive $t=0 \mathrm{np}$-pairing phase has yet been found. In addition, it was suggested that rotational properties (moments of inertia, band crossing frequencies etc) can provide a signal for the existence of isoscalar $n p$-pair condensate $9|22| 23|24| 25]$. These properties will be in the focus of the present manuscript.

In general case, the isovector and isoscalar $n p$-pairing as well as isospin symmetry conservation have to be taken into account in the $N \approx Z$ nuclei (see Ref. 27 and references quoted therein). On the mean field level, the symmetry breaking in the case of $n p$-pairing (especially of its isoscalar component because of the uncertainty with its strength) and isospin 28 can be small. In such situation, the exact methods of symmetry restoration by projection techniques have to be employed. Unfortunately, none of available theoretical tools take into account these correlations and requirements simultaneously reflecting the fact that such theories are extremely complicated. In particular, the isospin symmetry restoration in the presence of the $n p$-pairing has been neglected in almost all theoretical studies of the $N \approx Z$ nuclei. It is reasonable to expect that because of the complexity of the problem, no theoretical model, which will fully take into account above mentioned requirements, will be available in foreseeable future.

In such situation, isovector mean field theory 1 is a reasonable approximation for the study of rotational properties of the $N \approx Z$ nuclei, see Sect. 2. The present manuscript is an extension of our earlier systematic study of rotating $N \approx Z$ nuclei published in Ref. ${ }^{10}$. Analysis of recent experimental data in ${ }^{72} \mathrm{Kr}$ and ${ }^{76} \mathrm{Sr}$ within the isovector mean field theory, combined systematic results on the $N \approx Z$ nuclei 
and the analysis of the expected situation in ${ }^{64} \mathrm{Ge}$ will be presented in Sects. 3 , 4 [5] and 6, respectively. Section 7 summarizes our main conclusions.

\section{Isovector mean field theory}

The isovector mean-field theory 1 is used for the study of rotating properties of the $N \approx Z$ nuclei in the present manuscript. This theory assumes that there is no isoscalar $n p$-pairing, but takes into account isovector $n p$-pairing and isospin symmetry conservation. The later feature, treated in strong coupling limit, is a clear advantage of this approach since it is ignored in other studies. An additional advantage is the fact that standard mean field models with only $t=1$ like-particle pairing can be employed. The basis modification of these theories lies in adding the isorotational energy term $T(T+1) / 2 \mathcal{J}_{\text {iso }}$ to the total energy. Since, however, all low-lying rotational bands in even-even $N=Z$ nuclei have isospin $T=0$, this term vanishes. On the level of accuracy of the standard mean-field calculations, the restoration of the isospin symmetry (which takes care of the $\mathrm{t}=1 \mathrm{np}$ pair field) changes only the energy of the $T=1$ states relative to the $T=0$ states 1 . With this in mind, the rotating properties were studied by means of the cranked Relativistic Hartree-Bogoliubov 32 33|34 (CRHB) theory.

At high spin, the impact of $t=1$ pairing is negligible and consequently it can be neglected. In such situation, the isospin broken at low spin by isovector pairing is conserved automatically 27. Thus, the high spin $(I \geq 15 \hbar)$ states are systematically studied by means of the cranked Nilsson-Strutinsky (CNS) 36|37/38 and the cranked Relativistic Mean Field (CRMF) 39/40/41 approaches, which assume zero pairing. The standard set of Nilsson parameters 36 is used in the CNS calculations. The CRMF and CRHB calculations have been performed with the NL3 parameterization of the RMF Lagrangian 42 which provides good description of nuclear properties throughout nuclear chart. The D1S Gogny force 43 and approximate particle number projection by means of the Lipkin-Nogami (LN) method have been used in the pairing channel of the CRHB theory.

In the calculations without pairing, the shorthand notation $[p, n]$ indicating the number $p(n)$ of occupied $g_{9 / 2}$ proton (neutron) orbitals is used for labeling of the configurations. In the cases when the holes in the $f_{7 / 2}$ subshell play a role, an extended shorthand notation $\left[\left(p_{h}\right) p,\left(n_{h}\right) n\right]$ with $p_{h}\left(n_{h}\right)$ being the number of proton (neutron) $f_{7 / 2}$ holes is used. The $3_{i}$ label is used for mixed low- $j N=3$ orbitals, where subscript $i$ indicates the position of the orbital within the specific signature group.

In a number of publications it has been suggested that rotational properties of the $N \approx Z$ nuclei can provide evidence for the presence of a $t=0 \mathrm{np}$ pair field $9|22| 23|24| 25$. However, the reasoning often ignored the considerable $\beta$ - and $\gamma$-softness of the nuclei in the mass region of interest $2|22| 23$. The question which physical observables of rotating nuclei may present evidence for the existence of the $t=0 n p$ pair field is addressed in the present manuscript. The size of the mo- 
4 Authors' Names

ment of inertia, the frequencies at which the pairs of particles align their angular momentum (band crossing frequencies), deformation properties, and unexpected mixing of configurations with a different number of quasiparticles have been discussed in the literature as possible indicators of $n p$-pairing in rotating $N \approx Z$ nuclei $119|22| 23|24| 25|26| 44$.

\section{3. ${ }^{72} \mathrm{Kr}$ nucleus}

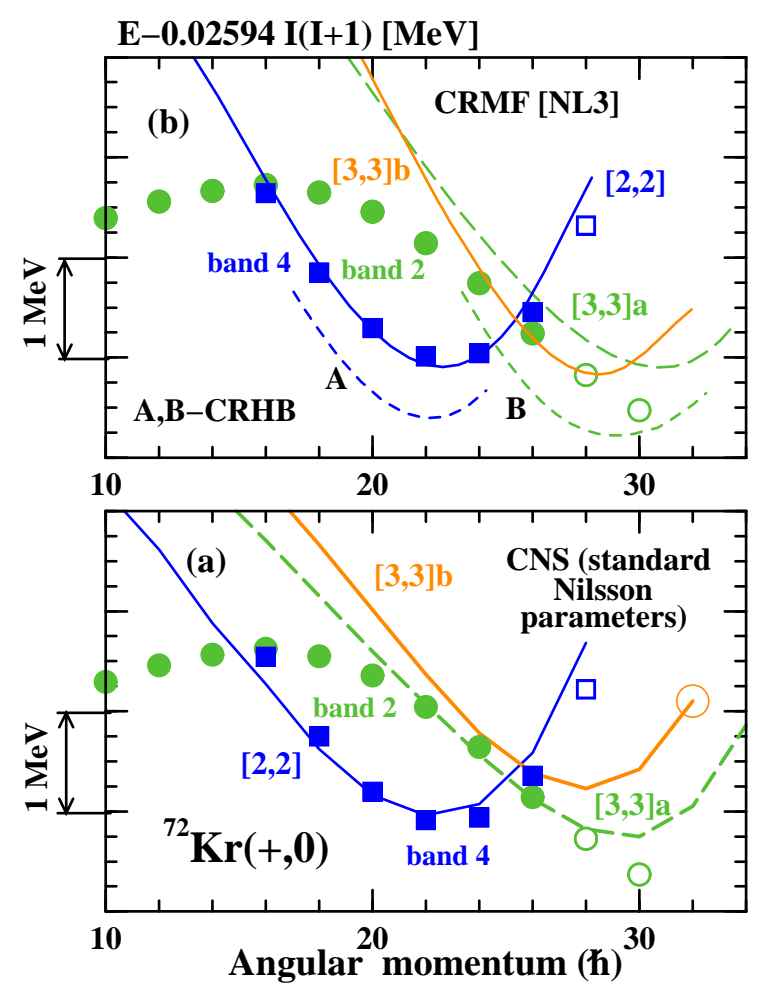

Fig. 1. Excitation energies of the experimental bands 2 and 4 in ${ }^{72} \mathrm{Kr}$ and theoretical configurations calculated in the CRMF, CRHB (panel (a)), and CNS (panel (b)) approaches relative to a rigid rotor reference $E_{R L D}=E-0.02594 I(I+1)$. Experimental data are shown by symbols, while lines are used for theoretical results. Open symbols are used for the states observed recently in Refs. $29 \mid 30$

In recent experiment 29130, previously observed bands were extended to an excitation energy of $\sim 24 \mathrm{MeV}$ and angular momentum of $30 \hbar$, new side band has been observed and the lifetimes of high-spin states were measured for the first time. These data allow to check further the accuracy of the description of rotating nuclei within the isovector mean field theory. In particular, it allow to see if there is any 
enhancement of the quadrupole deformation in the $N=Z$ nuclei. Ref. 45 predicted that the $t=0 n p$-pairing generates such enhancement. An important aspect of this study is the fact that all theoretical calculations (partially published in Ref. 10; see also Ref. 31 for the results of the CNS calculations employing different set of model parameters) were performed before the data became available, and, thus they can be considered as predictions.

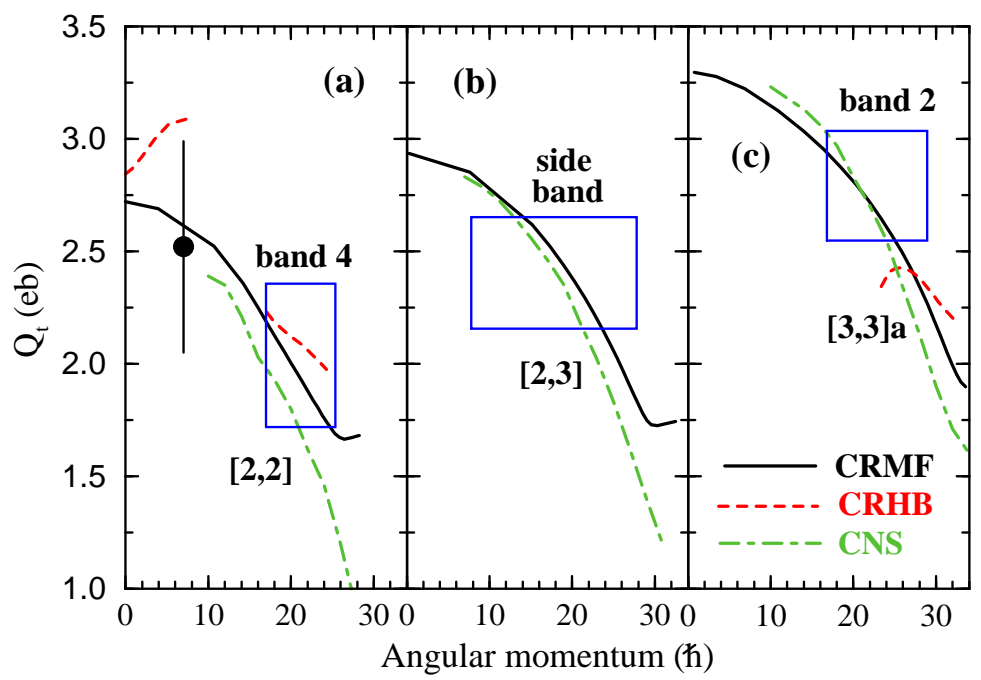

Fig. 2. Transition quadrupole moments as a function of angular momentum. The data point at $I=7 \hbar$ is from Ref. 35 , while boxes represent the measured transition quadrupole moments and their uncertainties within the measured spin range from the present work. The results of the CRMF and CRHB calculations are shown by solid and long-dashed lines, respectively.

Fig. [1 shows the experimental excitation energies minus a rigid rotor reference versus angular momentum for bands 2 and 4 and the corresponding theoretical configurations. In Ref. 10 band 4 was assigned to the $[2,2]$ configuration (i. e. the double S-band). This band (including recently observed $I=28 \hbar$ state) is well described by the CNS and CRMF calculations. These calculations also indicate the presence of two closely lying [3,3] configurations (Fig. 1), which are the candidates for the band 2. The configurations $[3,3] \mathrm{a}$ and $[3,3] \mathrm{b}$ are obtained from the $[2,2]$ configuration by exciting a proton and a neutron from the $3_{3}(\alpha=-1 / 2)$ and $3_{3}(\alpha=+1 / 2)$ orbitals into second $g_{9 / 2}(\alpha=+1 / 2)$ orbital, respectively. The details of the interpretation of band 2 are, however, model dependent reflecting the fact that the description of the energies of the single-particle states is not optimal (see Refs. 38146). The CNS calculations with the Nilsson parameters from Ref. 47 ('A80' parameters) and the CRMF calculations are similar and they suggest that the band 2 may be the envelope of the [3,3] a and [3,3]b configurations (see top panel in Fig. 
11), whereas the CNS calculations with the standard Nilsson parameters suggest the $[3,3]$ a configuration. In the former case the irregularities seen in $J^{(2)}$ of the band B at $\omega \geq 0.8 \mathrm{MeV}$ (see Fig. 2 in Ref. 18 ) may be explained as due to the crossing (or interaction) of the $[3,3] \mathrm{a}$ and $[3,3] \mathrm{b}$ configurations.

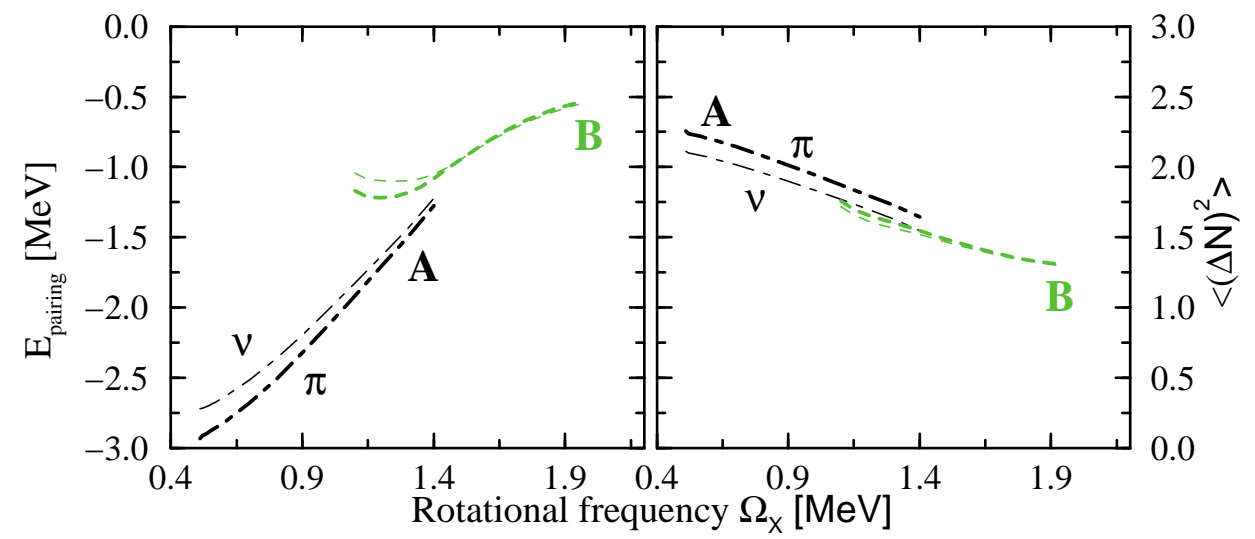

Fig. 3. Calculated values of the pairing energies $E_{\text {pairing }}=-1 / 2 \operatorname{Tr}(\Delta \kappa)$ and particle number fluctuations $\left\langle(\Delta N)^{2}>\right.$ as a function of rotational frequency $\Omega_{X}$ in the CRHB configurations A and $\mathrm{B}$ shown in Fig. 1 The notation of the lines is given in the figure.

However, a number of factors favor the assignment of the [3,3] a configuration to the band 2. An analysis of the relative energies of experimental high-spin bands in ${ }^{73,74} \mathrm{Kr}$ and ${ }^{70} \mathrm{Br}$ shows that they are better described in the CNS calculations with the standard set of the Nilsson parameters as compared with the ones employing 'A80' parameters. The experimental $E-E_{R L D}$ plot at spin larger than $20 \hbar$ is better described by the [3,3] a configuration (see Fig. (1). The transition quadrupole moment $Q_{t}$ of the configuration $[3,3] \mathrm{b}$ is smaller than the one of the $[3,3] \mathrm{a}$ configuration by $0.5-0.75 \mathrm{eb}$ in the spin range of interest (see Fig. 11 in Ref. 10 ). While the [3,3]a configuration reproduces the observed values of $Q_{t}$ of band 2 reasonably well (Fig. 21), the same will not be possible if the configuration [3,3]b is assigned to band 2 .

The CRHB calculations were performed for the configurations A and B which are the paired analogs of unpaired $[2,2]$ and $[3,3]$ a configurations (Fig. 11). Their energies are lower than those of their unpaired analogs by approximately $0.7 \mathrm{MeV}$. The pairing correlations in these configurations are small (see Fig. 3) and comparable with the ones in the SD band of ${ }^{60} \mathrm{Zn}$ above the paired band crossing ${ }^{53}$. They decrease with increasing rotational frequency reflecting the Coriolis anti-pairing effect. As a consequence of weak pairing correlations, the results of the CRHB calculations are very close to the ones of CRMF for the physical observables of interest such as $\left(E-E_{R L D}\right)$ plots (Fig. 11) (and, as a result, kinematic and dynamic 
moments of inertia), and transition quadrupole moments (Fig. 2). It is, however, necessary to recognize that due to the deficiences of the Lipkin-Nogami method in the regime of weak pairing 54 the CRMF calculations without pairing can be better approximation to exact solution at medium and high spins than those within the CRHB+LN framework 34 .

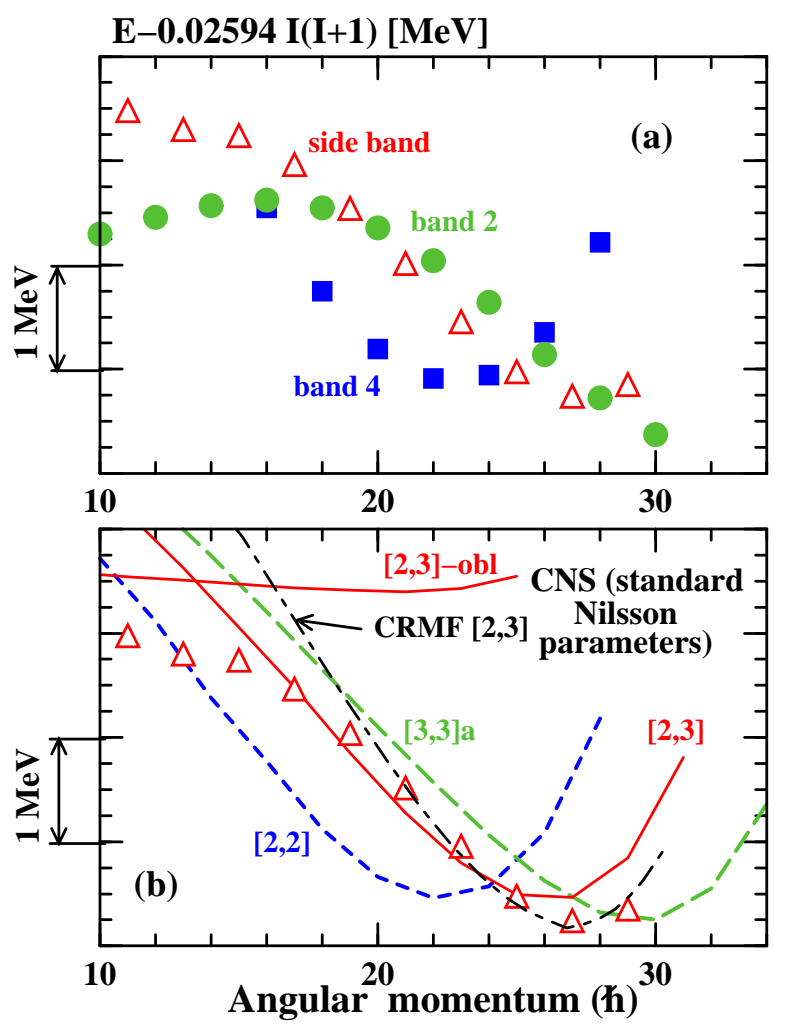

Fig. 4. The same as Fig. 1 but for the side band. Panel (a) shows experimental bands, while the results of the CNS calculations for their theoretical counterparts are given in panel (b). In addition, the CRMF $[2,3]$ configuration is shown in panel (b) by dash-dotted line.

Side band has been observed in Ref. 29|30. It is linked to the ground state band by the 1685 and $1653 \mathrm{keV}$ transitions of unknown multipolarity. If one assumes E2 multipolarity for these transitions, then this band would have parity $\pi=+$ and signature $r=0$. With this assignment, all observed high-spin bands would have the same parity-signature contrary to theoretical results obtained in the CNS and CRMF calculations which suggest the presence of near-yrast rotational sequences of negative parity (see Fig. 4). Thus, E1 multipolarity is more likely choice for the multipolarities of these transitions. With this assignment, side band has negative 
parity and extends from spin $7^{-}$up to spin $29^{-}$. As follows from the $E-E_{R L D}$ plot of this band, it is built from 3 configurations with configuration changes (band crossings) taking place at $I=11 \hbar$ and $I=17 \hbar$. The high spin branch, which starts at $I=17 \hbar$, is well described by the $[2,3]$ configuration in the CNS and CRMF calculations (Fig. 4). At high spin, the relative energies of the band 4, side band and band 2 are well described in the CNS calculations with the standard Nilsson parameters by the $[2,2],[2,3]$ and $[3,3]$ configurations (see Fig. 44). The calculations also suggest that low spin branch of side band may be associated with oblate '[2,3]obl' configuration (Fig. 4).

Fig. 2 compares measured transition quadrupole moments of observed bands with the ones of assigned configurations. Starting from the $[2,2]$ configuration (band 4 ), subsequent additions of the $g_{9 / 2}$ particle(s) increase the transition quadrupole moment. This trend is seen both in calculations and in experiment. In addition, absolute values of $Q_{t}$ are well described in the calculations. Experimental data on transition quadrupole moments are also available for ${ }^{73,74} \mathrm{Kr}{ }^{55 \mid 56}$ and ${ }^{74} \mathrm{Rb} 55$. These data (both absolute values and relative changes in $Q_{t}$ ) agree reasonably well with the results of the CNS, CRMF, and CRHB calculations (see Refs. 10|30|55|56 for details). In addition, available data on transition quadrupole moments of superdeformed rotational bands in ${ }^{59} \mathrm{Cu}{ }^{51}$ and ${ }^{60} \mathrm{Zn} 49$ are well reproduced in similar calculations. Thus, one can conclude that no enhancement of quadrupole deformation in the $N=Z$ nuclei (which is expected in the presence of the $t=0$ np-pairing $45)$ as compared with the one obtained within the framework of isovector mean field theory is required in order to reproduce experiment.

\section{4. ${ }^{76} \mathrm{Sr}$ nucleus: probing Coulomb antipairing effect.}

The progress in understanding of $n p$-pairing requires better knowledge of different components of like-particle pairing. The investigation of the impact of the Coulomb exchange term on the pairing field, within the framework of the Hartree-FockBogoliubov approach based on the Gogny force, found a considerable decrease of the proton pairing energies due to a Coulomb anti-pairing effect 57 . Recent experimental data on ${ }^{76} \mathrm{Sr} \frac{52}{}$, when combined with the limited results from previous high spin studies of other $A=58-80 N=Z$ nuclei in which $g_{9 / 2}$ proton/ neutron

paired band crossings have been observed $\left({ }^{60} \mathrm{Zn} 58,{ }^{68} \mathrm{Se} 59,{ }^{72} \mathrm{Kr}{ }^{60}\right)$ provide us with the first real opportunity to test these predictions 52 .

The similarity of the proton and neutron single-particle spectra (apart from some constant shift in absolute energies by the Coulomb energy) in the $N=Z$ nuclei leads to the fact that proton and neutron pairing energies are almost the same for proton and neutron subsystems in calculations which do not contain a Coulomb exchange term (as is the case with CRHB calculations, see Fig. 3). As a consequence, the alignment (paired band crossing) of proton and neutron pairs takes place at the same rotational frequency in such calculations (see Fig. (5)), which in turn leads to only one bump in the dynamic moment of inertia. However, if 


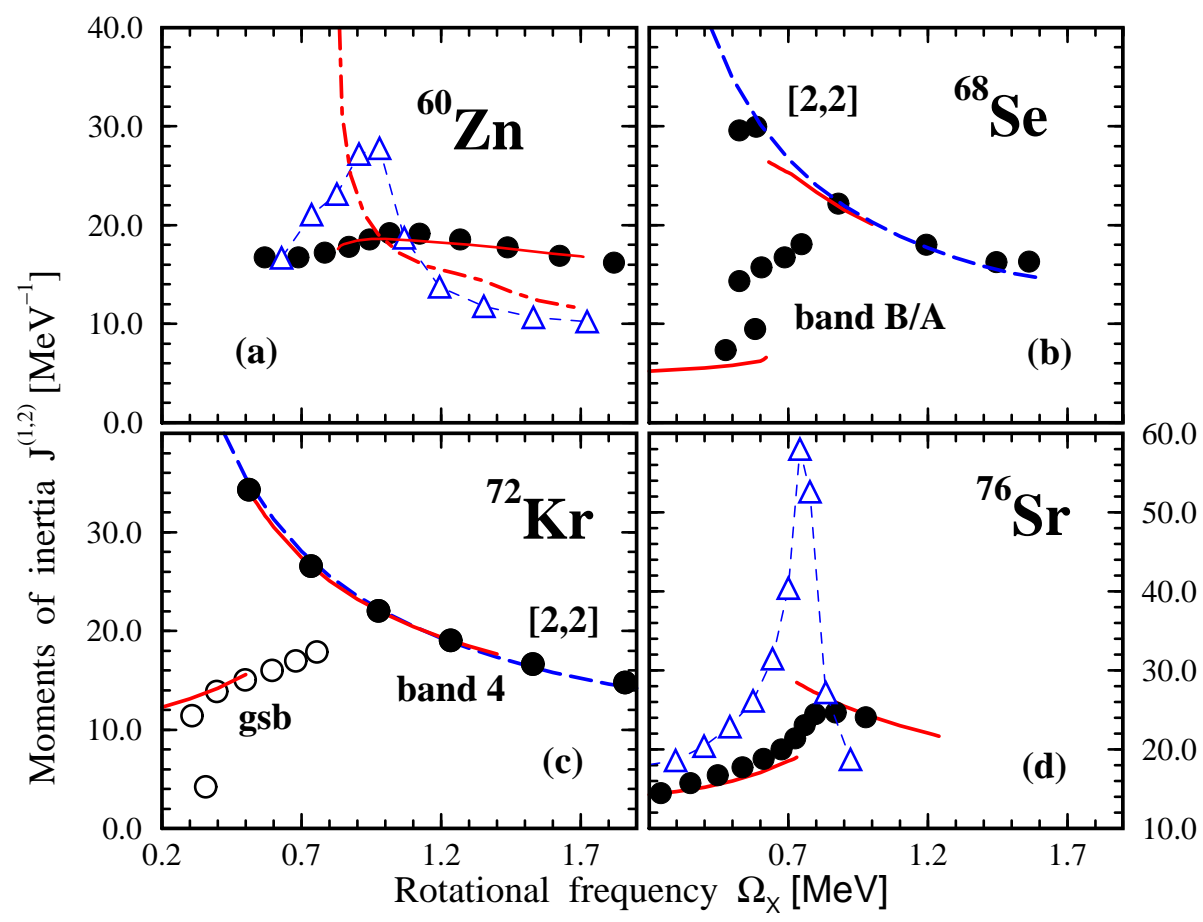

Fig. 5. Kinematic and dynamic moments of inertia of rotational bands in even-even $N=Z$ nuclei. The dynamic moments of inertia (open triangles) are shown only for ${ }^{60} \mathrm{Zn}$ and ${ }^{76} \mathrm{Sr}$. The results of the CRHB calculations are shown by solid and dash-dotted lines for kinematic and dynamic moments of inertia, respectively. The kinematic moments of inertia obtained in the CRMF calculations are shown by dashed lines in ${ }^{68}$ Se and ${ }^{72} \mathrm{Kr}$.

the predictions of Ref. 57 are correct then the proton pairing energy should be considerably smaller than that due to the neutrons, and it is reasonable to expect that this fact will result in an alignment of proton and neutron pairs at different frequencies, which would manifest itself in a double peaked shape for the dynamic moments of inertia.

Fig. [5]shows the kinematic moments of inertia for all four nuclei and the dynamic moments of inertia for ${ }^{60} \mathrm{Zn}$ and ${ }^{76} \mathrm{Sr}$. The ground and $I^{\pi}=2^{+}$states of ${ }^{68} \mathrm{Se}$ and ${ }^{72} \mathrm{Kr}$ are believed to be oblate (see Ref. 10) which leads to low values for the kinematic moments of inertia at low frequencies (see Fig. 5). With increasing spin highly-triaxial $\left({ }^{68} \mathrm{Se}\right)$ or near-prolate $\left({ }^{72} \mathrm{Kr}\right)$ structures become yrast ${ }^{10}$. Thus, the first irregularity seen in the kinematic moments of inertia of these nuclei at a rotational frequency $\hbar \omega \sim 0.4 \mathrm{MeV}$ is due to this shape coexistence. However such shape coexistence is not present in ${ }^{60} \mathrm{Zn}$ and ${ }^{76} \mathrm{Sr}$ at low spin. These nuclei are characterized by gradually increasing kinematic moments of inertia at low rotational frequency (see Fig. 5). In ${ }^{68} \mathrm{Se}$ the band crossing seen at $\hbar \omega \sim 0.7 \mathrm{MeV}$ is not 


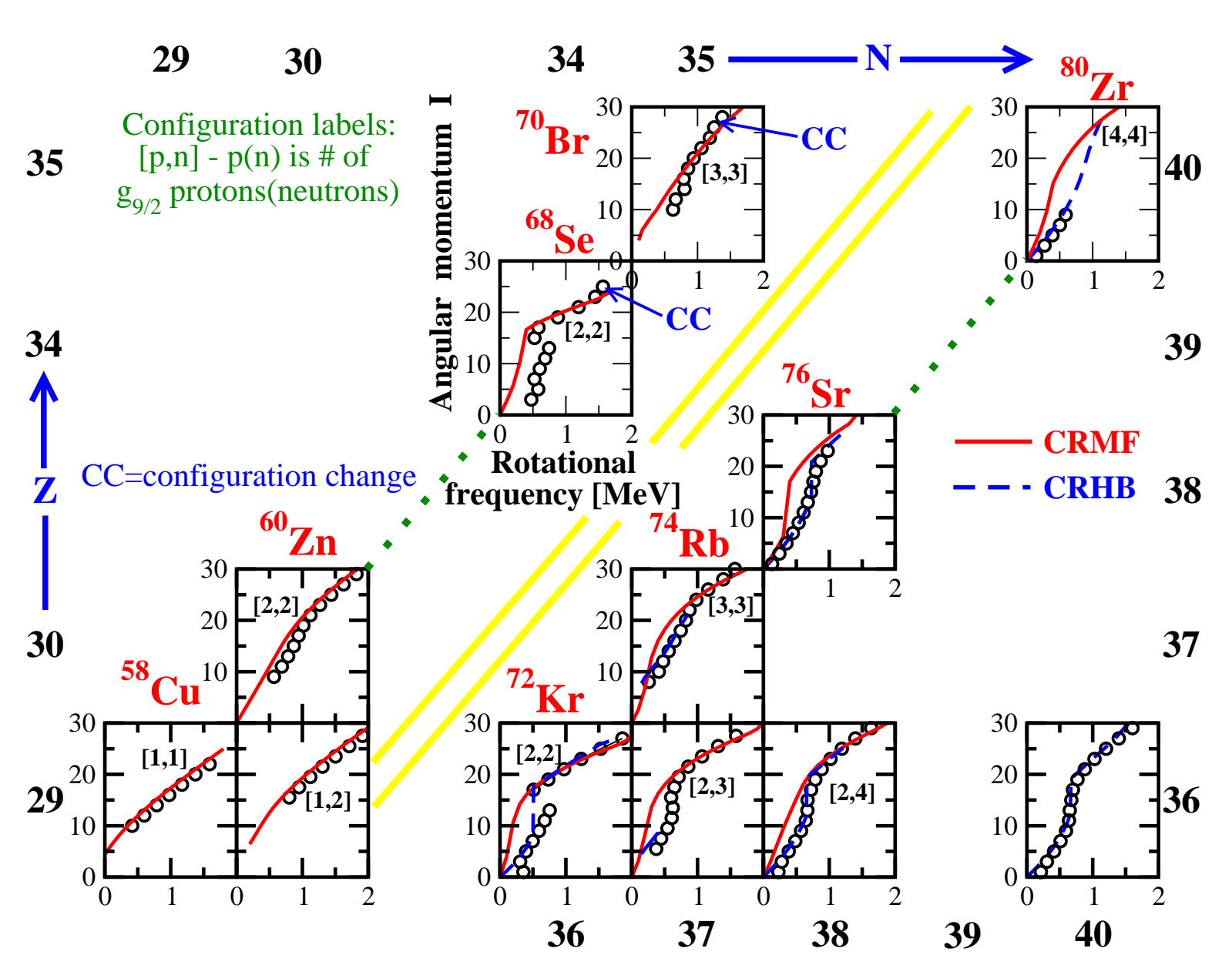


related to the standard change from the ground $(g-)$ band to the $g_{9 / 2}$ aligned proton/ neutron $S$-band 10 , and, thus, can be excluded from consideration. It is clear, however, that for the other three nuclei the proton and neutron $g_{9 / 2}$ paired band crossings take place simultaneously at $\hbar \omega=0.6-1.0 \mathrm{MeV}$. As a result, the currently available experimental data in even-even $N=Z$ nuclei do not support the existence of the Coulomb anti-pairing effect caused by the Coulomb exchange term. These data are also well described in the CRHB and CRMF (above paired band crossing) calculations (see Fig. 5 and Refs. $\underline{10 / 52 / 53}$ for details).

\section{Systematics of rotational and deformation properties}

The experimental data on alignments in rotational structures of the $N \approx Z$ nuclei are compared with the results of the CRMF and CRHB calculations in Fig. 6 , One can see good agreement between experiment and the CRMF calculations at high spin as well as between experiment and CRHB calculations at low spin. In addition, experimental data on transition quadrupole moments are available for ${ }^{59} \mathrm{Cu}{ }^{51},{ }^{60} \mathrm{Zn} 49,{ }^{72,73,74} \mathrm{Kr}{ }^{29|30| 55 \mid 56}$ and ${ }^{74} \mathrm{Rb} 55$. These data agree well with the results of the CNS, CRMF, and CRHB calculations (see Sect. 3 in the present manuscript and Refs. $10|30| 49|51| 55 \mid 56$ for details).

\section{6. ${ }^{64} \mathrm{Ge}$ nucleus}

As follows from Fig. 6. the knowledge of the $N=Z{ }^{62} \mathrm{Ga},{ }^{64} \mathrm{Ge},{ }^{66} \mathrm{As}$, and ${ }^{78} \mathrm{Yb}$ nuclei is restricted to low-spin states. The CNS calculations for ${ }^{64} \mathrm{Ge}$ are performed in order to better understand what can be expected at high spin in these nuclei.

It has been pointed out before that ${ }^{64} \mathrm{Ge}$ nucleus is soft with respect to $\gamma-$ and octupole deformations (see Ref. 61 and references quoted therein). Fig. 7 shows the results of the CNS calculations (which are restricted to reflection symmetric shapes), which also indicate softness toward $\gamma$-deformation. Indeed, the $[0,0](\alpha=0)$ configuration is characterized by the $\left(\varepsilon_{2} \sim 0.2, \gamma \sim-30^{\circ}\right)$ deformation in the spin range $I=2-8 \hbar$. The yrast lines of other combinations of parity and signature are characterized by similar deformation in the spin range $I=0-3 \hbar$. Up to spins $I \sim 35 \hbar$, the yrast lines are dominated by the states with the deformations $\varepsilon_{2} \approx 0.25-0.35$ and $\gamma=26^{\circ}-60^{\circ}$. The terminating bands, many of which terminate in a favored way 38 , dominate the yrast region up to $I \sim 35 \hbar$. Superdeformed bands with deformation $\varepsilon_{2} \sim 0.5, \gamma \sim 10^{\circ}$ become yrast above that spin.

It seems that the complicated structure of this nucleus, dominated in the spin region of interest by $\gamma-$ (and probably octupole) softness and terminating structures [which are not that different from the ones in ${ }^{68} \mathrm{Se}$ (see Fig. 7 in Ref. ${ }^{10}$ ) and

in ${ }^{74} \mathrm{Kr}$ (see Fig. 3 in ${ }^{10}$ )], will not allow to obtain reliable evidences of the isoscalar $t=0 n p$-pairing even if the experimental data will be extended to higher spin. 


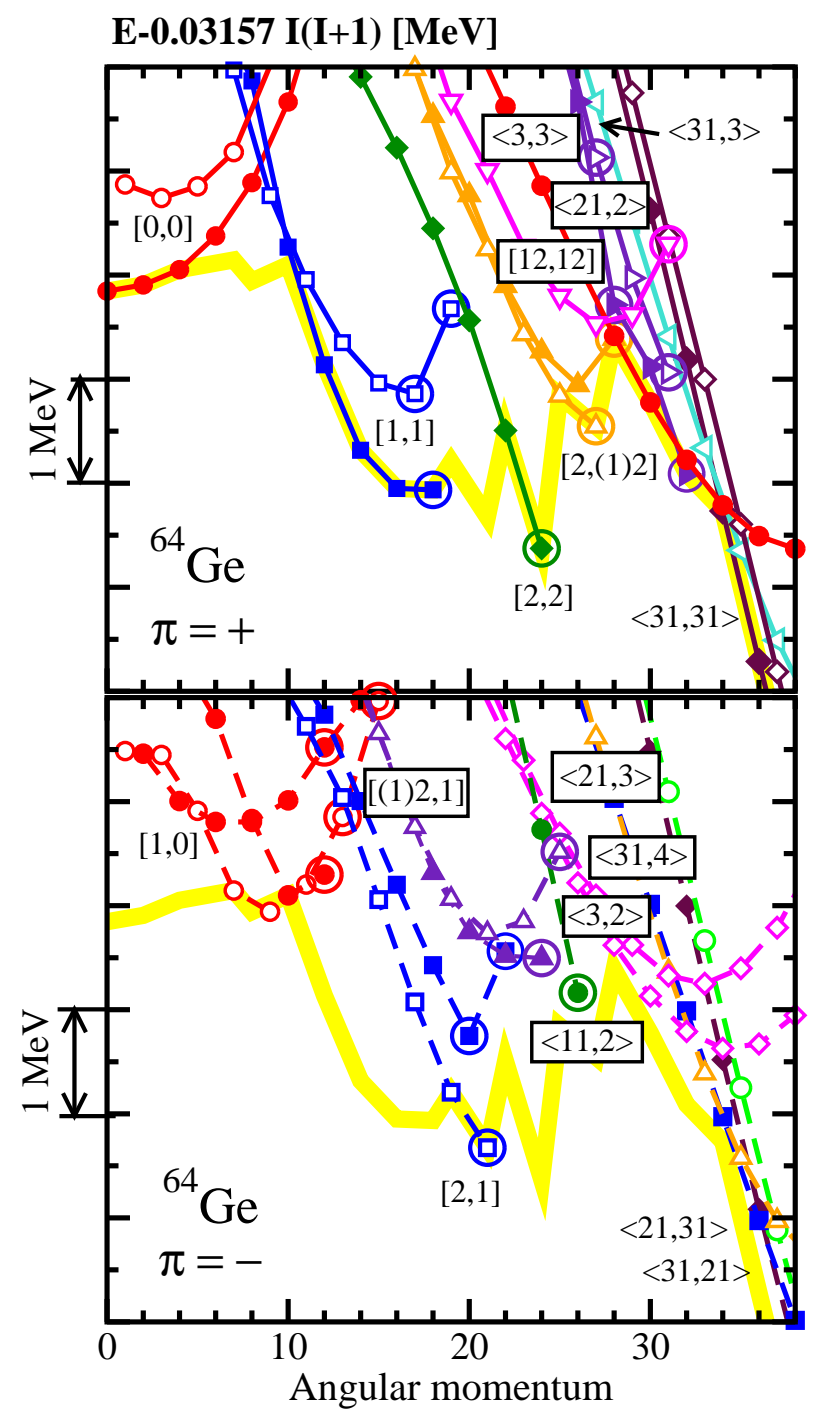

Fig. 7. Excitation energies of the configurations forming the yrast lines of 4 combinations of parity and signature in ${ }^{64} \mathrm{Ge}$ given relative to a rigid rotor reference $E_{R L D}=0.03157 I(I+1) \mathrm{MeV}$. Calculated terminating (aligned) states are encircled. The shorthand notation $\left\langle p_{1} p_{2}, n_{1} n_{2}\right\rangle$ indicates the number $p_{1}\left(n_{1}\right)$ of occupied $g_{9 / 2}$ proton (neutron) orbitals and the number $p_{2}\left(n_{2}\right)$ of occupied $h_{11 / 2}$ proton (neutron) orbitals. $p_{2}\left(n_{2}\right)$ are omitted when later orbitals are not occupied. Wide line indicate the total yrast line. The same type of symbols is used for signature partner orbitals. Solid (open) symbols are used for $\alpha=0(1)$ configurations. 


\section{Conclusions}

The systematic analysis of the rotational response and deformation properties of the $N \approx Z$ nuclei support the interpretation of these nuclei within the isovector mean field theory. According to this framework, there is no isoscalar $n p$ pair field. At low spin, strong isovector pair field exists, which includes a large $n p$ component, the strength of which is determined by isospin conservation. Like in nuclei away from the $N=Z$ line, this isovector pair field is destroyed by rotation. In this high spin regime the calculations without pairing describe well the data provided the drastic shape changes that cause among other things band termination are taken into account. No clear evidence for the existence of the isoscalar $t=0 \mathrm{np}$ pairing has been found. However, due to limitations of our theoretical tools one cannot completely exclude the possibility of the existence of $n p$ pairing condensate in the $t=0$ channel or the possibility that the rotational properties are not sensitive to this type of pairing.

\section{Acknowledgements}

The work was supported by the DOE grant DE-FG02-07ER41459. I would like to express my gratitude to S. Frauendorf, R. Wadsworth, and C. Andreoiu for their contributions to this project.

\section{References}

1. S. G. Frauendorf and J. A. Sheikh, Nucl. Phys. A645, 509 (1999).

2. A. O. Macchiavelli, P. Fallon, R. M. Clark, M. Cromaz, M. A. Deleplanque, R. M. Diamond, G. J. Lane, I. Y. Lee, F. S. Stephens, C. E. Svensson, K. Vetter, and D. Ward, Phys. Rev. C61, 041303(R) (2000).

3. P. Vogel, Nucl. Phys. A662, 148 (2000).

4. C. D. O'Leary, C. E. Svensson, S. G. Frauendorf, A. V. Afanasjev, D. E. Appelbe, R. A. E. Austin, G. C. Ball, J. A. Cameron, R. M. Clark, M. Cromaz, P. Fallon, D. F. Hodgson, N. S. Kelsall, A. O. Macchiavelli, I. Ragnarsson, D. Sarantites, J. C. Waddington and R. Wadsworth, Phys. Rev. C67, 021301(R) (2003).

5. D. R. Bes, R. A. Broglia, O. Hansen, O. Nathan, Phys. Rep. 34, 1 (1977).

6. A. O. Macchiavelli, P. Fallon, R. M. Clark, M. Cromaz, M. A. Deleplanque, R. M. Diamond, G. J. Lane, I. Y. Lee, F. S. Stephens, C. E. Svensson, K. Vetter, and D. Ward, Phys. Lett. B480, 1 (2000).

7. E. Garrido, P. Sarriguren, E. Moya de Guerra, U. Lombardo, P. Schuck, H. J. Schulze, Phys. Rev. C63, 037304 (2001).

8. D. C. Zheng and L. Zamick, Ann. Phys. (N.Y.) 206, 106 (1991).

9. S. Satuła and R. Wyss, Phys. Lett. B393, 1 (1997).

10. A. V. Afanasjev and S. Frauendorf, Phys. Rev. C71, 064318 (2005).

11. K. Muto, E. Bender, and H. V. Klapdor, Z. Phys. A334, 47 (1989).

12. J. Engel, S. Pittel, M. Stoitsov, P. Vogel, and J. Dukelsky, Phys. Rev. C55, 1781 (1997).

13. P. Möller, B. Pfeiffer, and K.-L. Kratz, Phys. Rev. C67, 055802 (2003).

14. T. Nikšić, T. Marketin, D. Vretenar, N. Paar, and P. Ring, Phys. Rev. C71, 014308 (2005). 
15. G. Pantis, F. Simkovic, J. D. Vergados, A. Faessler, Phys. Rev. C53, 695 (1996).

16. O. Civitarese, P. O. Hess, J. G. Hirsch, and M. Reboiro, Phys. Rev. C59, 194 (1999).

17. P. Fröbrich, Z. Phys. 236, 153 (1970).

18. P. Fröbrich, Phys. Lett. B37, 338 (1971).

19. S. Głowacz, W. Satuła, and R. A. Wyss, Eur. Phys. J. A19, 33 (2004).

20. G. Röpke, A. Schnell, P. Schuck, and P. Nozières, Phys. Rev. Lett. 80, 3177 (1998).

21. M. Hasegawa and K. Kaneko, Phys. Rev. C61, 037306 (2000).

22. K. Kaneko and J. Zhang, Phys. Rev. C57, 1732 (1998).

23. J. A. Sheikh and R. Wyss, Phys. Rev. C62, 051302(R) (2000).

24. S. Satuła and R. Wyss, Nucl. Phys. A676, 120 (2000).

25. A. L. Goodman, Phys. Rev. C63, 044325 (1999).

26. N. S. Kelsall, S. M. Fischer, D. P. Balamuth, G. C. Ball, M. P. Carpenter, R. M. Clark, J. Durell, P. Fallon, S. J. Freeman, P. A. Hausladen, R. V. F. Janssens, D. G. Jenkins, M. J. Leddy, C. J. Lister, A. O. Macchiavelli, D. G. Sarantites, D. C. Schmidt, D. Seweryniak, C. E. Svensson, B. J. Varley, S. Vincent, R. Wadsworth, A. N. Wilson, A. V. Afanasjev, S. Frauendorf, I. Ragnarsson and R. Wyss, Phys. Rev. C65, 044331 (2002).

27. A. L. Goodman, Phys. Rev. C60, 014311 (1999).

28. J. Dobaczewski and I. Hamamoto, Phys. Lett. B345, 181 (1995).

29. C. Andreoiu, C. E. Svensson, R. A. E. Austin, M. P. Carpenter, D. Dashdorj, P. Finlay, S. J. Freeman, P. E. Garrett, A. Görgen, J. Greene, G. F. Grinyer, B. Hyland, D. Jenkins, F. Johnston-Theasby, P. Joshi, A. O. Macchiavelli, F. Moore, G. Mukherjee, A. A. Phillips, W. Reviol, D. G. Sarantites, M. A. Schumaker, D. Seweryniak, M. B. Smith, J. J. Valiente-Dobon, and R. Wadsworth, Phys. Scripta T125, 127 (2006).

30. C. Andreoiu, C. E. Svensson, A. V. Afanasjev, R. A. E. Austin, M. P. Carpenter, D. Dashdorj, P. Finlay, S. J. Freeman, P. E. Garrett, J. Greene, G. F. Grinyer, A. Görgen, B. Hyland, D. Jenkins, F. Johnston-Theasby, P. Joshi, A. O. Machiavelli, F. Moore, G. Mukherjee, A. A. Phillips, W. Reviol, D. G. Sarantites, M. A. Schumaker, D. Seweryniak, M. B. Smith, J.J. Valiente-Dobón, R. Wadsworth, submitted to Phys. Rev. $C$

31. B. G. Carlsson and I. Ragnarsson, American Institute of Physics, Conference Proceedings 831, Int. Conference "Frontiers in Nuclear Structure, Astrophysics, and Reactions: FINUSTAR", edited by S. V. Harissopulos, P. Demetriou, and R. Julin, (2006) p. 60.

32. A. V. Afanasjev, J. König, and P. Ring, Phys. Rev. C60, 051303 (1999).

33. A. V. Afanasjev, P. Ring, and J. König, Nucl. Phys. A676, 196 (2000).

34. D. Vretenar, A. V. Afanasjev, G. Lalazissis, and P. Ring, Phys. Rep. 409, 101 (2005).

35. G. de Angelis, C. Fahlander, A. Gadea, E. Farnea, W. Gelletly, A. Aprahamian, D. Bazzacco, F. Becker, P. G. Bizzeti, A. Bizzeti-Sona, F. Brandolini, D. de Acuña, M. De Poli, J. Eberth, D. Foltescu, S. M. Lenzi, S. Lunardi, T. Martinez, D. R. Napoli, P. Pavan, C. M. Petrache, C. Rossi Alvarez, D. Rudolph, B. Rubio, W. Satuła, S. Skoda, P. Spolaore, H. G. Thomas, C. A. Ur, and R. Wyss, Phys. Lett. B415, 217 (1997).

36. T. Bengtsson and I. Ragnarsson, Nucl. Phys. A436, 14 (1985).

37. A. V. Afanasjev and I. Ragnarsson, Nucl. Phys. A591, 387 (1995).

38. A. V. Afanasjev, D. B. Fossan, G. J. Lane and I. Ragnarsson, Phys. Rep. 322, 1 (1999).

39. W. Koepf and P. Ring, Nucl. Phys. A493, 61 (1989).

40. J. König and P. Ring, Phys. Rev. Lett. 71, 3079 (1993).

41. A. V. Afanasjev, J. König and P. Ring, Nucl. Phys. A608, 107 (1996).

42. G. A. Lalazissis, J. König and P. Ring, Phys. Rev. C55, 540 (1997).

43. J. F. Berger, M. Girod, and D. Gogny, Comp. Phys. Comm. 63, 365 (1991). 
44. D. G. Jenkins, N. S. Kelsall, C. J. Lister, D. P. Balamuth, M. P. Carpenter, T. A. Sienko, S. M. Fischer, R. M. Clark, P. Fallon, A. Görgen, A. O. Macchiavelli, C. E. Svensson, R. Wadsworth, W. Reviol, D. G. Sarantites, G. C. Ball, J. Rikovska Stone, O. Juillet, P. van Isacker, A. V. Afanasjev and S. Frauendorf, Phys. Rev. C65, 064307 (2002).

45. J. Terasaki, R. Wyss, and P.-H. Heenen, Phys. Lett. B437, 1 (1998).

46. A. V. Afanasjev, T. L. Khoo, S. Frauendorf, G. A. Lalazissis, and I. Ahmad, Phys. Rev. C67, 024309 (2003).

47. D. Galeriu, D. Bucurescu, and M. Ivaşku, J. Phys. G 12, 329 (1986).

48. S. M. Fischer, C. J. Lister, D. P. Balamuth, R. Bauer, J. A. Becker, L. A. Bernstein, M. P. Carpenter, J. Durell, N. Fotiades, S. J. Freeman, P. E. Garrett, P. A. Hausladen, R. V. F. Janssens, D. Jenkins, M. Leddy, J. Ressler, J. Schwartz, D. Svelnys, D. G. Sarantites, D. Seweryniak, B. J. Varley, and R. Wyss, Phys. Rev. Lett. 87, 132501 (2001).

49. A. V. Afanasjev, I. Ragnarsson and P. Ring, Phys. Rev. C59, 3166 (1999).

50. N. S. Kelsall, S. M. Fischer, D. P. Balamuth, G. C. Ball, M. P. Carpenter, R. M. Clark, J. Durell, P. Fallon, S. J. Freeman, P. A. Hausladen, R. V. F. Janssens, D. G. Jenkins, M. J. Leddy, C. J. Lister, A. O. Macchiavelli, D. G. Sarantites, D. C. Schmidt, D. Seweryniak, C. E. Svensson, B. J. Varley, S. Vincent, R. Wadsworth, A. N. Wilson, A. V. Afanasjev, S. Frauendorf, I. Ragnarsson and R. Wyss, Phys. Rev. C65, 044331 (2002).

51. C. Andreoiu, D. Rudolph, C. E. Svensson, A. V. Afanasjev, J. Dobaczewski, I. Ragnarsson, C. Baktash, J. Eberth, C. Fahlander, D. S. Haslip, D. R. LaFosse, S. D. Paul, D. G. Sarantites, H. G. Thomas, J. C. Waddington, W. Weintraub, J. N. Wilsson and C.-H. Yu, Phys. Rev. C62, 051301(R) (2000).

52. P. J. Davies, A. V. Afanasjev, R. Wadsworth, C. Andreoiu, R. A. E. Austin, M. P. Carpenter, D. Dashdorj, S. J. Freeman, P. E. Garrett, A. Görgen, J. Greene, D. G. Jenkins, F. L. Johnston-Theasby, P. Joshi, A. O. Macchiavelli, F. Moore, G. Mukherjee, W. Reviol, D. Sarantites, D. Seweryniak, M. B. Smith, C. E. Svensson, J. J. ValienteDobon, D. Ward, submitted to Phys. Rev. C.

53. A. V. Afanasjev, P. Ring and I. Ragnarsson, Proc. Int. Workshop PINGST2000 "Selected topics on $N=Z$ nuclei", 2000, Lund, Sweden, Eds. D. Rudolph and M. Hellström, (2000) p. 183.

54. J. A. Sheikh, P. Ring, and R. Rossignoli, Phys. Rev. C66, 044318 (2006).

55. F. Johnston-Theasby et al, in preparation, to be submitted to Phys. Rev. C.

56. J. J. Valiente-Dobón, T. Steinhardt, C. E. Svensson, I. Ragnarsson, A. V. Afanasjev, C. Andreoiu, R. A. E. Austin, M. P. Carpenter, D. Dashdorj, G. de Angelis, F. Dönau, J. Eberth, E. Farnea, P. Finlay, S. J. Freeman, A. Gadea, P. E. Garrett, A. Görgen, G. F. Grinyer, B. Hyland, D. Jenkins, F. Johnston-Theasby, P. Joshi, A. Jungclaus, K. P. Lieb, A. O. Macchiavelli, F. Moore, G. Mukherjee, D. R. Napoli, A. A. Phillips, C. Plettner, W. Reviol, D. Sarantites, H. Schnare, M. A. Schumaker, R. Schwengner, D. Seweryniak, M. B. Smith, I. Stefanescu, O. Thelen, R. Wadsworth, D. Ward, Phys. Rev. Lett. 95, 232501 (2005).

57. M. Anguiano, J. L. Egido and L. M. Robledo, Nucl. Phys. A683, 227 (2001).

58. C. E. Svensson, D. Rudolph, C. Baktash, M. A. Bentley, J. A. Cameron, M. P. Carpenter, M. Devlin, J. Eberth, S. Flibotte, A. Galindo-Uribarri, G. Hackman, D. S. Haslip, R. V. F. Janssens, D. R. LaFosse, T. J. Lampman, I. Y. Lee, F. Lerma, A. O. Macchiavelli, J. M. Nieminen, S. D. Paul, D. C. Radford, P. Reiter, L. L. Riedinger, D. G. Sarantites, B. Schaly, D. Seweryniak, O. Thelen, H. G. Thomas, J. C. Waddington, D. Ward, W. Weintraub, J. N. Wilson, C. H. Yu, A. V. Afanasjev, and I. Ragnarsson, 
Phys. Rev. Lett., 82, 3400 (1999).

59. S. M. Fischer, C. J. Lister and D. P. Balamuth, Phys. Rev. C67, 064318 (2003).

60. N. S. Kelsall, C. E. Svensson, S. Fischer, D. E. Appelbe, R. A. E. Austin, D. P. Balamuth, G. C. Ball, J. A. Cameron, M. P. Carpenter, R. M. Clark, M. Cromaz, M. A. Deleplanque, R. M. Diamond, J. L. Durell, P. Fallon, S. J. Freeman, P. A. Hausladen, D. F. Hodgson, R. V. F. Janssens, D. G. Jenkins, G. J. Lane, M. J. Leddy, C. J. Lister, A. O. Macchiavelli, C. D. O'Leary, D. G. Sarantites, F. S. Stephens, D. C. Schmidt, D. Seweryniak, B. J. Varley, S. Vincent, K. Vetter, J. C. Waddington, R. Wadsworth, D. Ward, A. N. Wilson, A. V. Afanasjev, S. Frauendorf, I. Ragnarsson and R. Wyss, Proc. Int. Conf. on "Frontiers of Nuclear Structure", (Berkeley, California, 2002), AIP Conf. Proc. v. 656, Eds. P. Fallon and R. Clark, (Melville, New York, 2003) p. 261.

61. P. J. Ennis, C. J. Lister, W. Gelletly, H. G. Price, B. J. Varley, P. A. Butler, T. Hoare, S. Cwiok, W. Nazarewicz, Nucl. Phys. A535, 392 (1991). 